\title{
Configurational entropy of magnetic skyrmions as an ideal gas
}

\author{
R. Zivieri, ${ }^{1, *}$ R. Tomasello, ${ }^{2, \dagger}$ O. Chubykalo-Fesenko, ${ }^{3}$ V. Tiberkevich, ${ }^{4}$ M. Carpentieri, ${ }^{5}$ and G. Finocchio ${ }^{1, \$}$ \\ ${ }^{1}$ Department of Mathematical and Computer Sciences, Physical Sciences and Earth Sciences, University of Messina, 98166 Messina, Italy \\ ${ }^{2}$ Institute of Applied and Computational Mathematics, Foundation for Research and Technology, GR 70013 Heraklion, Crete, Greece \\ ${ }^{3}$ Instituto de Ciencia de Materiales de Madrid, CSIC, Cantoblanco, 28049 Madrid, Spain \\ ${ }^{4}$ Department of Physics, Oakland University, Rochester, Michigan 48309, USA \\ ${ }^{5}$ Department of Electrical and Information Engineering, Politecnico di Bari, 70125 Bari, Italy
}

(Received 12 March 2019; revised manuscript received 14 May 2019; published 30 May 2019; corrected 11 July 2019)

\begin{abstract}
The study of thermodynamics of topological defects is an important challenge to understand their underlying physics. Among them, magnetic skyrmions have a leading role for their physical properties and potential applications in storage and neuromorphic computing. In this paper, the thermodynamic statistics of magnetic skyrmions is derived. It is shown that the skyrmion free energy can be modeled via a parabolic function and the diameters statistics obeys the Maxwell-Boltzmann distribution. This allows for making an analogy between the behavior of the distribution of skyrmion diameters statistics and the diluted gas Maxwell-Boltzmann molecules distribution of velocities at thermodynamic equilibrium. The calculation of the skyrmion configurational entropy, due to thermally induced changes of size and shape of the skyrmion, is essential for the determination of thermal fluctuations of the skyrmion energy around its average value. These results can be employed to advance the field of skyrmionics.
\end{abstract}

DOI: 10.1103/PhysRevB.99.174440

\section{INTRODUCTION}

Magnetic skyrmions have been gaining an important role in studies of low-dimensional magnetic systems due to their suitable physical properties and potential applications [1-4]. Skyrmions can be considered as quasiparticles with topologically protected magnetization texture, characterized by an integer skyrmion number $[1,4]$. Although skyrmions can be stabilized by the interplay between exchange and dipolar interactions (so-called "bubble skyrmion" [1]), much of the interest is devoted to systems where the DzyaloshinskiiMoriya interaction (DMI) plays a role in this stabilization $[5,6]$. The DMI is a chiral exchange interaction due to lack or breaking of inversion symmetry in bulk crystalline lattices (bulk DMI) [7-9] or at the interfaces in magnetic multilayers [interfacial DMI (IDMI)] [10-13]. While other types of DMI can exist (for instance, in $D_{2 d}$ structures) [14,15], in this work we focus on the IDMI. This because it promotes the formation of small Néel skyrmions [1,2,4], which are stable at room temperature as isolated skyrmions, and can be nucleated [16-18], manipulated [12,19-21], as well as detected [22-24] by electrical currents. Therefore, Néel skyrmions have become promising for technological applications [25-33]. Fundamentally, because of thermal fluctuations, Néel skyrmions are subject to (i) internal deformations $[10,12,34,35]$ that are responsible for the loss of the circular symmetry; (ii) thermal drift $[18,34,35]$, which leads to a random skyrmion motion throughout the film plane; and (iii) thermal

\footnotetext{
*zivieri@fe.infn.it

†rtomasello@iacm.forth.gr

‡gfinocchio@unime.it
}

breathing modes [35-37] that can induce non-stationary expansion and shrinking of the skyrmion core, i.e., a time evolution of the skyrmion size. Hence, the effect of thermal fluctuations should be considered for a proper design of skyrmionbased devices and applications [32,35], especially at room temperature.

In this work, we show that the thermal fluctuations promote the existence of a number of skyrmions characterized by the same energy, but having different shapes and diameters. This aspect allows for the definition of a skyrmion configurational entropy by using a statistical thermodynamic analogy between the skyrmion diameters population and the noninteracting molecules of an ideal gas [38,39]. This approach is based on the analytical formulation previously developed [35] considering a region of parameters where the two following hypotheses are verified: (i) the skyrmion energy can be well approximated by a square function of the skyrmion diameter near the minimum, and (ii) the skyrmion diameters distribution is well described by a Maxwell-Boltzmann (MB) function. The validity of those two hypotheses is checked by taking advantage of full micromagnetic simulations for different combinations of temperature and external field. Note that, in principle, also a third hypothesis according to which the skyrmion does not annihilate should be taken into account. However, for the range of magnetic parameters used and the range of temperatures investigated, we have found that the skyrmion has a long enough lifetime (500 ns) in the micromagnetic simulations and, therefore, does not annihilate. The skyrmion average diameter and its standard deviation, as well as the skyrmion entropy, can be analytically derived. In addition, the developed model can also be extended to the description of further magnetic textures, such as bubbles and vortices. 


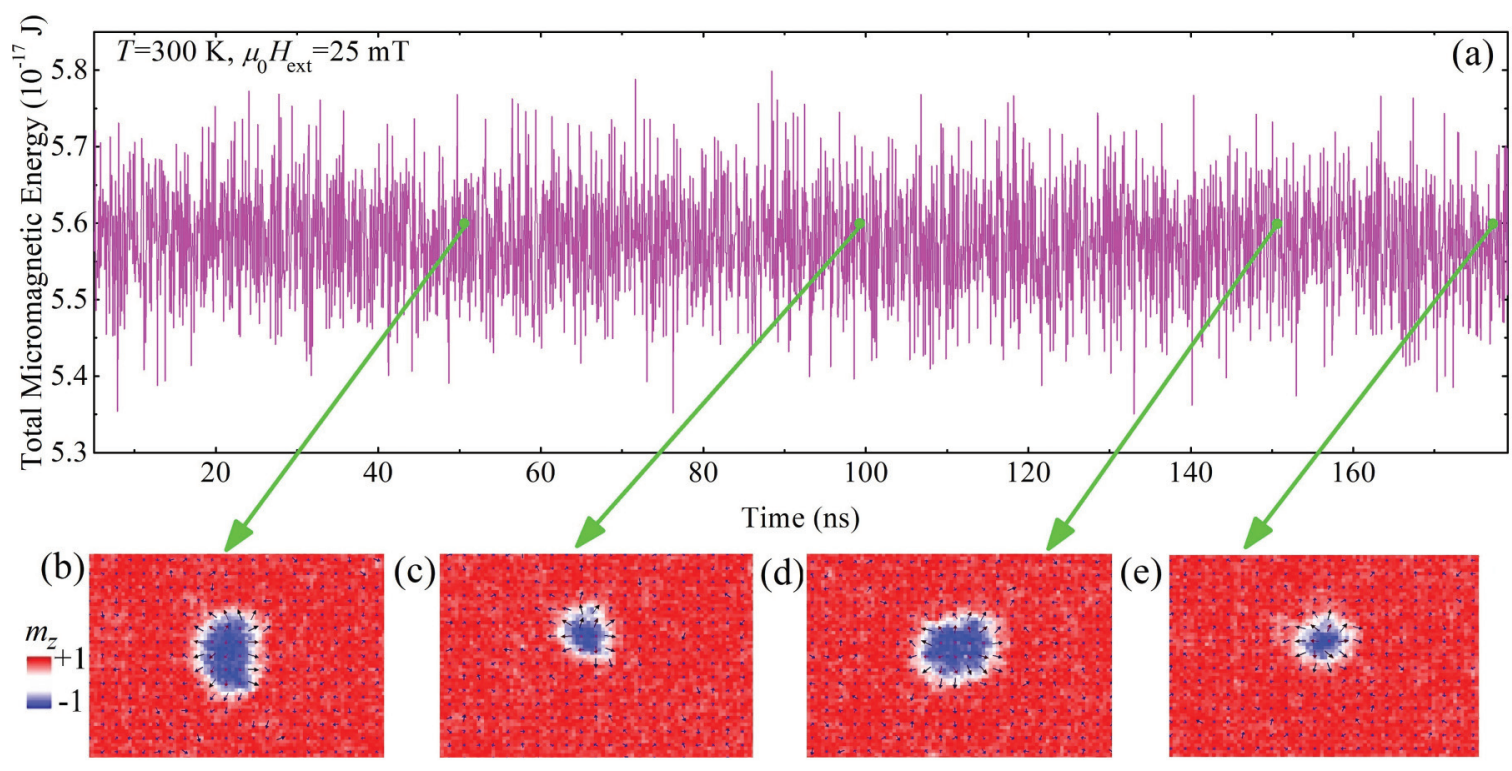

FIG. 1. (a) Total micromagnetic energy as a function of time for one of the simulations $\left(T=300 \mathrm{~K}\right.$, and $\left.\mu_{0} H_{\text {ext }}=25 \mathrm{mT}\right)$. The four green circles indicate an example of time instants at which different skyrmions have the same energy. (b)-(e) Snapshots of the skyrmion at the time instants indicated in (a). The arrows and background colors are linked to the $z$ component of the magnetization. The colors of the arrows indicate a positive (blue), negative (red), and zero (black) $z$ component, while the color bar refers to the background colors, as shown in the figure.

From a theoretical point of view, the knowledge of the skyrmion configurational entropy allows us to construct the proper thermodynamics for determining the fluctuations for many important quantities, such as the free energy. We show that a distribution of skyrmion diameters can be described in a canonical ensemble that is equivalent to a microcanonical ensemble because fluctuations of energy are much smaller than the skyrmion average energy.

Until now, the skyrmion entropy has been estimated from the experimental data, and it has been used to characterize the type (first- or second order) of magnetization phase transition around the transition temperature in bulk B20 compounds [40,41], and as a corrective term to the Arrhenius law, to explain the discrepancy between experimental and theoretical calculations of the lifetime of a skyrmion lattice [42]. However, no direct dependence of the entropy on the physical parameters, temperature, and geometrical characteristics of the skyrmion has been expressed. This work will fill this gap, giving a simple analytical model to be used as a support for the experimental works at finite temperatures.

The paper is organized as follows. In Sec. II the numerical model is briefly outlined. Section III presents the analytical model describing the calculation of the skyrmion energy and of the skyrmion diameters distribution, the average skyrmion diameter, the standard deviation, the configurational entropy, and the energy fluctuations. In Sec. IV conclusions are drawn.

\section{NUMERICAL MODEL}

We start by performing micromagnetic simulations at finite temperature to generate the data to be analyzed. We consider a circular nanodot of diameter $2 R_{d}=400 \mathrm{~nm}$ of a ferromagnetic material (we consider cobalt here) with a thickness $t=0.8 \mathrm{~nm}$ assumed to be coupled with a thin layer of heavy metal giving a sufficiently large IDMI, i.e., platinum. We perform systematic micromagnetic simulations to calculate the skyrmion sizes as a function of the out-ofplane external field $\mu_{0} H_{\text {ext }}$ and temperature $T$, by integrating the Landau-Lifshitz-Gilbert (LLG) equation for the reduced magnetization $\mathbf{m}=\mathbf{M} / M_{s}$ [43-49] $\left(M_{\mathrm{s}}\right.$ is the saturation magnetization; see note 1 in the Supplemental Material [43]). At $T=0 \mathrm{~K}$, we used the following material parameters: $M_{\mathrm{S}}=$ $600 \mathrm{kA} / \mathrm{m}$ [12], exchange stiffness constant $A=20 \mathrm{pJ} / \mathrm{m}$ [50], IDMI parameter $D=3.0 \mathrm{~mJ} / \mathrm{m}^{2}$ [51,52], perpendicular uniaxial anisotropy constant $K_{\mathrm{u}}=0.60 \mathrm{MJ} / \mathrm{m}^{3}$ [12,53], and Gilbert damping $\alpha_{\mathrm{G}}=0.1$ [54], while for $T>0$ and in the analytical model, we used the parameters as calculated from the scaling relations [35] $A(m)=A(T=0 \mathrm{~K}) m(T)^{1.5}, D(T)=$ $D(T=0 \mathrm{~K}) m(T)^{1.5}$, and $K_{\mathrm{u}}(T)=K_{\mathrm{u}}(T=0 \mathrm{~K}) m(T)^{3.6}$.

For the micromagnetic simulations, the thermal effects are included in the LLG equation as a stochastic term $\mathbf{h}_{\mathrm{th}}$ added to the deterministic effective magnetic field in each computational cell $\mathbf{h}_{\mathrm{th}}=\left(\chi / M_{\mathrm{s}}\right) \sqrt{2\left(\alpha_{\mathrm{G}} k_{\mathrm{B}} T / \mu_{0} \gamma_{0} \Delta V M_{\mathrm{s}} \Delta t\right)}$, with $k_{\mathrm{B}}=1.38 \times 10^{-23} \mathrm{~J} / \mathrm{K}$ the Boltzmann constant, $\mu_{0}$ the vacuum permeability, $\gamma_{0}$ the gyromagnetic ratio, $\Delta V$ the volume of the computational cell, $\Delta t$ the simulation time step, and $\chi$ a three-dimensional (3D) white Gaussian noise with zero mean and unit variance $[48,49]$. The thermal fields, in each computational cell, are uncorrelated. The discretization cell size used is $2.5 \times 2.5 \times 0.8 \mathrm{~nm}^{3}$ [43] (see note 1 in the Supplemental Material for more details).

The effective diameter is calculated by assuming that the area of the skyrmion core (here it is the region where the $z$ component of the magnetization is negative) is equivalent to a circle [55].

Figure 1(a) shows the time dependence of the total micromagnetic energy $E[\mathbf{m}]=\int d V \varepsilon(\mathbf{m})$ of the ferromagnet $[\varepsilon(\mathbf{m})$ is the energy density] as calculated by micromagnetic 
simulations from the spatial distribution of the magnetization m:

$$
\begin{aligned}
\varepsilon(\mathbf{m})= & A(\nabla \mathbf{m})^{2}+D\left[m_{z}(\nabla \cdot \mathbf{m})-(\mathbf{m} \cdot \nabla) m_{z}\right]+K_{\mathrm{u}}\left(1-m_{z}^{2}\right) \\
& -0.5 M_{\mathrm{s}} \mu_{0} \mathbf{m} \cdot \mathbf{H}_{\mathrm{m}}-M_{\mathrm{s}} \mu_{0} \mathbf{m} \cdot \mathbf{H}_{\mathrm{ext}},
\end{aligned}
$$

where $m_{z}$ is the $z$ component of the magnetization, $\mathbf{H}_{\mathrm{m}}$ is the magnetostatic field, and $\mathbf{H}_{\text {ext }}$ is the external magnetic field.

It can be observed that there exist skyrmion configurations with different shape and size [Figs. 1(b)-1(e)], but characterized by the same energy $\left[5.6 \times 10^{-17} \mathrm{~J}\right.$ in Fig. 1(a)]. These results suggest that a skyrmion configurational entropy can be introduced and regarded as the number of different skyrmions having the same energy.

\section{ANALYTICAL MODEL}

\section{A. Skyrmion energy}

The computation of the configurational entropy is based on the determination of the skyrmion energy.

The skyrmion magnetization texture in cylindrical coordinates can be written in the form $\mathbf{m}(x, y)=$ $\sin \theta(\rho) \cos \phi_{0} \hat{\rho}+\sin \theta(\rho) \sin \phi_{0} \hat{\phi}+\cos \theta(\rho) \hat{z}$, where $\theta(\rho)$ is the radially dependent magnetization distribution angle and $\phi_{0}$ is an azimuthal angle. Setting $\phi_{0}=0$, we get the outwardly directed radial Néel skyrmion texture, $\mathbf{m}(x, y)=\sin \theta(\rho) \hat{\rho}+\cos \theta(\rho) \hat{z}$. In the present study, we have considered an outwardly radial Néel skyrmion with a negative core characterized by a skyrmion number $Q=-1$, a cylindrical symmetry with respect to the out-of-plane direction ( $z$ axis), and it is stabilized in a magnetic circular dot. The results derived for Néel skyrmion can indeed be generalized to other magnetic textures.

The skyrmion energy $E$ is calculated as a volume integral of the skyrmion energy density $E\left(r_{\text {sky }}\right)=\int \varepsilon\left(r, r_{\text {sky }}\right) d V=$ $\int_{-t / 2}^{t / 2} d z \int_{0}^{2 \pi} d \phi \int_{0}^{R_{d}} \varepsilon\left(r, r_{\text {sky }}\right) \rho d \rho$. For the Néel skyrmion, we use the following trial equilibrium magnetization distribution through the ansatz $\theta_{0}(r)=2 \arctan \left[\frac{r_{\text {sky }}}{r} e^{\xi\left(r_{\text {sky }}-r\right)}\right]$, with $r_{\text {sky }}=R_{\text {sky }} / l_{\text {exch }}$ the dimensionless skyrmion radius, $l_{\text {exch }}=$ $\sqrt{2 A / \mu_{0} M_{\mathrm{s}}{ }^{2}}$ is the exchange length, with $A$ the material exchange stiffness, $t$ the dot thickness, and $r=\rho / l_{\text {exch }}[35,56]$. This skyrmion ansatz has been previously shown to have an excellent agreement with direct micromagnetic simulations [35,57]. The skyrmion energy density $\varepsilon=\varepsilon_{\text {exch }}+\varepsilon_{\text {IDMI }}+$ $\varepsilon_{\text {ani }}+\varepsilon_{\text {ext }}$ contains all the relevant contributions. In particular, $\varepsilon_{\text {exch }}=A(\nabla \mathbf{m})^{2}$ is the exchange energy density, $\varepsilon_{\text {IDMI }}=$ $|D|\left[m_{z}(\nabla \cdot \mathbf{m})-(\mathbf{m} \cdot \nabla) m_{z}\right]$ is the IDMI energy density and $D$ the DMI strength, $\varepsilon_{\text {ani }}=K_{\mathrm{u}}\left(1-m_{z}^{2}\right)+\frac{1}{2} \mu_{0} M_{\mathrm{s}}^{2} m_{z}^{2}$ is the anisotropy energy density with $K_{\mathrm{u}}$ the uniaxial anisotropy constant, and $\varepsilon_{\mathrm{ext}}=-\mu_{0} M_{\mathrm{s}} H_{\mathrm{ext}} m_{z}$ is the Zeeman energy density, with $\mu_{0} H_{\text {ext }}$ the amplitude of the external bias parallel to the $z$ axis. The magnetic parameters $A, D$, and $K_{\mathrm{u}}$ at nonzero temperature are scaled from their zero-temperature values, by using the scaling laws shown in Sec. II.

The first hypothesis of our analytical approach is that the skyrmion energy near the minimum can be described via a parabolic potential of the form $E_{\text {sky }} \simeq a\left(D_{\text {sky }}-D_{0 \text { sky }}\right)^{2}+b$. In the parabolic potential, the coefficient $a\left(\mathrm{~J} / \mathrm{m}^{2}\right)$ is proportional to the parabola curvature, $a=1 / 2 d^{2} E_{\text {sky }} / d D_{\text {sky }}{ }^{2}, D_{\text {sky }}$ is the generic skyrmion diameter ranging from 0 to $2 R_{d}$,
$D_{0 \text { sky }}$ is the equilibrium diameter corresponding to the energy minimum $E_{\mathrm{sky}}^{\min }$ for every $T$, and $b[\mathrm{~J}]$ is $b=E_{\mathrm{sky}}^{\min }$. The equilibrium skyrmion diameter depends on the temperature via the scaled values of the magnetic parameters; therefore, both $a$ and $b$ are temperature-dependent coefficients, $a(T)$ and $b(T)$. Figure 2(a) shows, in fact, how the coefficient $a$ changes with temperature, for three values of the external field. The general trend is a linear dependence of $a$ on the temperature and $a$ decreases with increasing temperature, marking a broadening of the potential well due to thermal effects. Moreover, at fixed temperature, $a$ increases with increasing the external-field amplitude indicating its narrowing effect on the potential well.

Figures 2(b) and 2(c) show that the parabolic curve fits well the analytical skyrmion energy when $0 \leqslant T \leqslant 200 \mathrm{~K}$ at zero external field, while at $T=300 \mathrm{~K}$ [Fig. 2(d)] the matching is less accurate. We ascribe this difference to the change of the energetic stability of the skyrmion when it is becoming the ground state (see Fig. 3 in Ref. [35]). In particular, for the parameters used in this study, the skyrmion is a metastable state when either $0 \leqslant T \leqslant 200 \mathrm{~K}$ for any external field in the range $0-50 \mathrm{mT}$, or $250 \leqslant T \leqslant 300 \mathrm{~K}$ only for $\mu_{0} H_{\mathrm{ext}}>5 \mathrm{mT}$, while it becomes stable outside these intervals. As was explained in Ref. [35] with details, during this transition from metastable to ground state the skyrmion radius is very sensitive to small variations of external parameters.

\section{B. Skyrmion diameters distribution}

The fact that the energy of the skyrmion is, as a first approximation, well described as a quadratic function of the skyrmion diameter leads us to suppose that the skyrmion diameters distribution can be treated as the distribution of particle velocities of an ideal gas, at least from a statistical thermodynamics viewpoint.

Therefore, the second step of our analytical approach is to check if the population of the skyrmion diameters follows a MB distribution function:

$$
\frac{d n}{d D_{\text {sky }}}=C_{\text {sky } 0} D_{\text {sky }}^{2} e^{-\left(a / k_{\mathrm{B}} T\right)\left(D_{\text {sky }}-D_{0 \text { sky }}\right)^{2}}
$$

where $d n$ is the number of times one gets a skyrmion diameter between $D_{\text {sky }}$ and $D_{\text {sky }}+d D_{\text {sky }}, C_{\text {sky } 0}$ is the normalization constant referred to the Gaussian distribution centered at the equilibrium skyrmion diameter $D_{0 \text { sky }}$ [43]. In this respect, note that, strictly speaking, the skyrmion structure is planar and thus the skyrmion distribution should be regarded as two dimensional while the ideal gas molecules MB velocity distribution is 3D. However, in micromagnetic simulations the thickness of the ferromagnetic material is taken into account. This observation allowed for considering approximately, also for the skyrmion diameters distribution, the 3D MB with the main difference that $D_{\text {sky }}$ is a scalar while the particle velocity is a vector. Note also that, analogously to the ideal gas, the skyrmion energy is comparable to the thermal energy in the range of temperatures $50-300 \mathrm{~K}$, thus ensuring a displacement effect of the maximum of the distribution as a function of temperature [38] (see note 2 in the Supplemental Material [43]). 

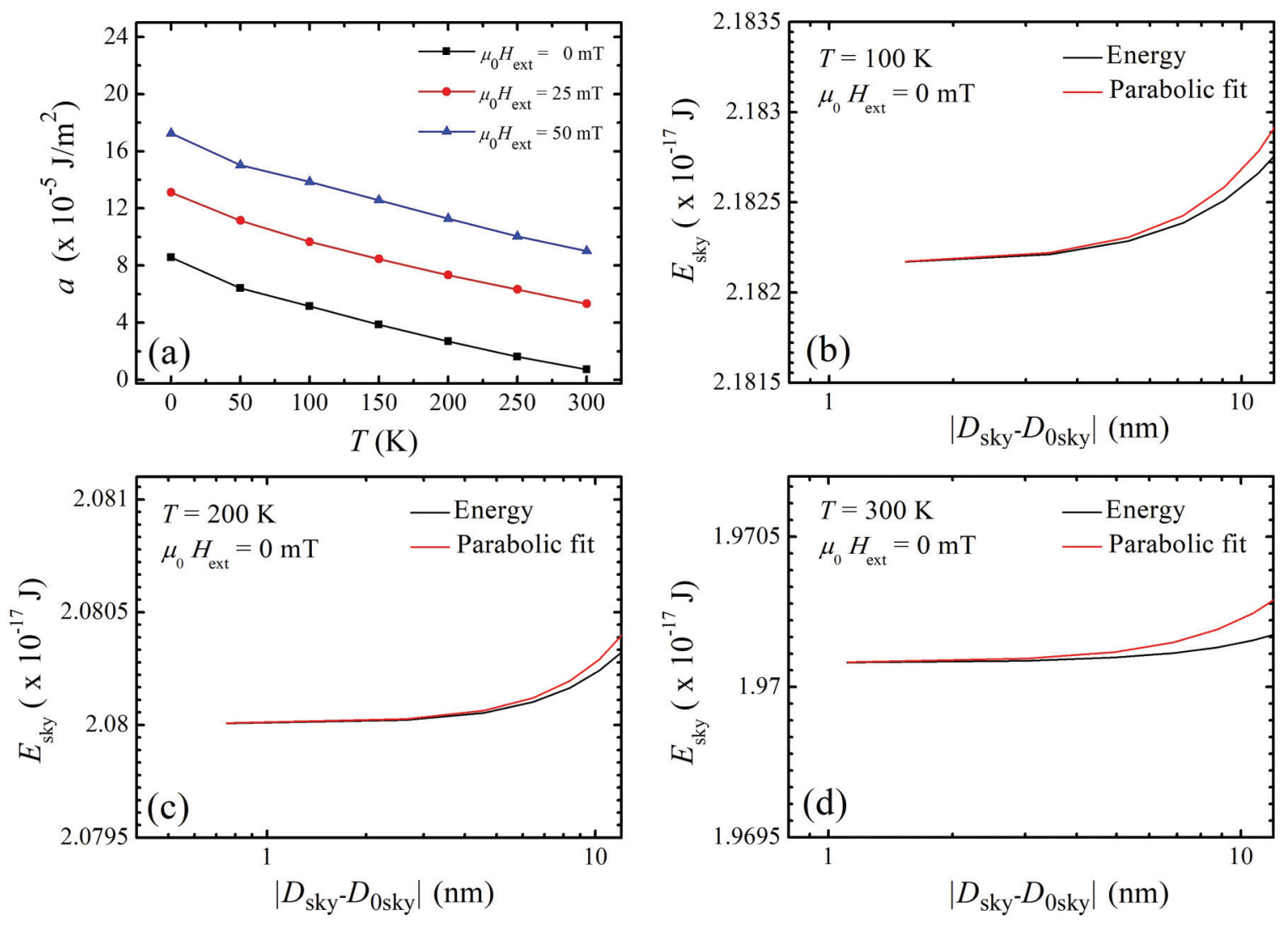

FIG. 2. (a) Fitting parameter $a$ as a function of $T$ for $\mu_{0} H_{\text {ext }}=0,25$, and $50 \mathrm{mT}$. Profile of the skyrmion energy close to the energy minimum (black curve) together with the parabolic fit (red curve) for $\mu_{0} H_{\text {ext }}=0 \mathrm{mT}$ and (b) $T=100 \mathrm{~K}$, (c) $T=200 \mathrm{~K}$, and (d) $T=300 \mathrm{~K}$.

Another analogy is that the number of noninteracting molecules of an ideal gas varies continuously as a function of velocity and possesses only translational kinetic energy, as the skyrmion diameters population referred to a single skyrmion, during thermal annealing, fluctuate independently along the radial direction, leading to continuous and infinitesimal changes of skyrmion size [see also Fig. 1(a)].

A further common property is represented by confinement. Like gas molecules obeying MB statistics are confined in a box, magnetic skyrmions of different diameters are formed in a confined magnetic system.

In order to prove this hypothesis, we compare the distribution of skyrmion diameters as computed from micromagnetic simulations and resulting from postprocessing to the analytical ones [Eq. (2)]. Figure 3 shows such a comparison at $T=100,200$, and $300 \mathrm{~K}$, respectively, for an applied field $\mu_{0} H_{\text {ext }}=25 \mathrm{mT}$. The agreement between the analytical and the micromagnetic results is excellent. Similar good agreements are also obtained for the other temperature/externalfield combinations in the region of metastability.

In order to confirm the very good agreement between the analytical distribution and the micromagnetic histograms, we compute the cumulative distribution function as the integral of the distributions. Figure 4(a) shows the results for 100, 200 , and $300 \mathrm{~K}$ when the applied external field is $25 \mathrm{mT}$, corresponding to the distributions of Fig. 3. The inset displays the root-mean-square deviation for the three cases, where the error is always smaller than $7 \%$. For this reason, we can consider that the agreement is excellent.
Since we have shown that the skyrmion energy can be considered as a quadratic function of the skyrmion diameter and that the skyrmion diameters population obeys the MB distribution, we can make the full analogy between the behavior of skyrmion diameters population and the velocity distribution of noninteracting molecules in an ideal gas. Let us examine this point in depth later in the text.

In Table I, we strengthen this analogy considering a skyrmion diameter statistics and an ideal gas statistics of $N$ noninteracting molecules at statistical thermodynamic equilibrium contained in a box that is described within a microcanonical ensemble where the total energy has a constant value independent of the time.

Strictly speaking, as observed in micromagnetic simulations, the total energy is not conserved in time so that the skyrmion diameters population exhibits fluctuations of energy and is in principle more properly described by a canonical ensemble. However, due to the high number of degrees of freedom represented by the skyrmion diameters population, we can suppose that the energy fluctuations are small (see Sec. III F for a quantitative estimation) regarding the ensemble as a microcanonical ensemble and therefore recovering the full analogy with the ideal gas statistics.

Looking at Table I, the skyrmion diameter $D_{\text {sky }}$ replaces the modulus of the velocity $v$ of the gas molecule, while the fitting parameter $a$, proportional to parabolic energy curvature, plays the same role as the constant $1 / 2 m$ depending on the mass $m$ of the molecule in the gas with the only difference that $a=a(T)$ and $m$ is a constant for a given gas. In this way, 

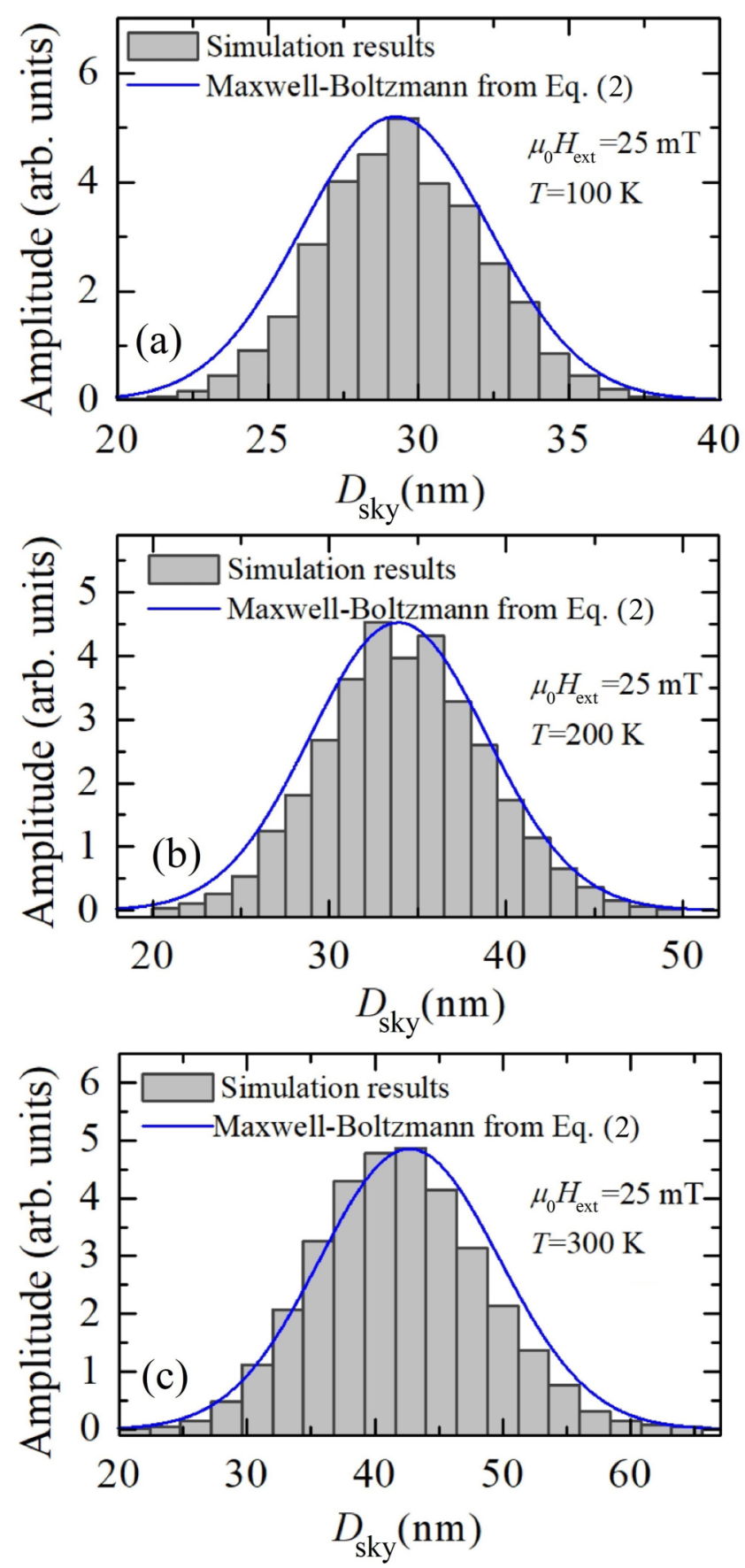

FIG. 3. Skyrmion diameters distribution for (a) $T=100 \mathrm{~K}$, (b) $T=200 \mathrm{~K}$, and (c) $T=300 \mathrm{~K}$ when $\mu_{0} H=25 \mathrm{mT}$. The histograms represent the results from micromagnetic simulations with thermal fields, while the blue curve is the analytical MB distribution as calculated from Eq. (2). In the analytical calculations, we used the scaled values of the magnetic parameters $A, D$, and $K_{\mathrm{u}}$ for each temperature [35] and obtained the following parameters: at $T=$ $100 \mathrm{~K}, a=9.67 \times 10^{-5} \mathrm{~J} / \mathrm{m}^{2}$ and $D_{0 \text { sky }}=26.83 \mathrm{~nm}$, at $T=200 \mathrm{~K}$, $a=7.33 \times 10^{-5} \mathrm{~J} / \mathrm{m}^{2}$ and $D_{0 \text { sky }}=32.03 \mathrm{~nm}$, and at $T=300 \mathrm{~K}$, $a=5.32 \times 10^{-5} \mathrm{~J} / \mathrm{m}^{2}$, and $D_{0 \text { sky }}=39.64 \mathrm{~nm}$.

the total skyrmion energy replaces the kinetic energy of a particle of the ideal gas and the MB distribution reproducing the statistical behavior of skyrmion diameters maps into the


FIG. 4. (a) Comparison between the analytical and micromagnetic cumulative distribution function (CDF) when $\mu_{0} H_{\mathrm{ext}}=25 \mathrm{mT}$. Inset: root-mean-square deviation (RMSD) as a function of temperature when $\mu_{0} H_{\mathrm{ext}}=25 \mathrm{mT}$. (b) Standard deviation as a function of temperature, for three values of the external field. The continuous lines with symbols represent the analytical calculations by means of Eq. (4), while the dashed lines with symbols indicate the results from micromagnetic simulations with thermal stochastic fields.

well-known MB distribution of velocities of $N$ noninteracting molecules in a diluted gas [38].

\section{Average skyrmion diameter}

Thanks to the aforementioned analogy, we can calculate the average skyrmion diameter $\left\langle D_{\text {sky }}(T)\right\rangle=$ $C_{\text {sky0 }} 2 \pi \int_{0}^{\infty} d D_{\text {sky }} D_{\text {sky }}^{3} e^{-\left(a / k_{B} T\right)\left(D_{\text {sky }}-D_{0 \text { sky }}\right)^{2}}$ in the same way as the average particle velocity in an ideal dilute gas at a given temperature and external bias field where $2 \pi$ comes from the integration over the polar angles $\theta$ and $\phi$ and $\quad C_{\text {sky } 0}=\left(2 \pi \int_{0}^{\infty} d D_{\text {sky }} D_{\text {sky }}^{2} e^{-\left(a / k_{B} T\right)\left(D_{\text {sky }}-D_{0 \text { sky }}\right)^{2}}\right)^{-1}$ is obtained after a normalization to unity of the MB distribution since we are considering one skyrmion exhibiting size variations in full analogy with the normalization to the number $N$ of noninteracting particles in an ideal gas. The integration yields

$$
\left\langle D_{\text {sky }}(T)\right\rangle \simeq D_{0 \text { sky }}\left(\frac{3 k_{\mathrm{B}} T+2 a D_{0 \text { sky }}^{2}}{k_{\mathrm{B}} T+2 a D_{0 \text { sky }}^{2}}\right),
$$

with $D_{0 \text { sky }}=D_{0 \text { sky }}(T)$ and $a=a(T)$. 
TABLE I. Analogy between an ideal gas of molecules and a magnetic skyrmion diameters population with $C_{\mathrm{g}}=4 \pi N\left(\mathrm{~m} /\left(2 \pi k_{\mathrm{B}} T\right)\right)^{3 / 2}$.

\begin{tabular}{lc}
\hline \hline Ideal gas & Skyrmion diameters \\
\hline$v$ & $D_{\text {sky }}$ \\
$1 / 2 m$ & $a$ \\
$\frac{d n}{d v}=C_{\mathrm{g}} v^{2} \exp \left(-1 / 2 m \mathrm{v}^{2} / k_{\mathrm{B}} T\right)$ & $\frac{d n}{d D_{\text {sky }}}=C_{\text {sky } 0} D_{\text {sky }}^{2} \exp \left(-a\left(D_{\text {sky }}-D_{0 \text { sky }}\right)^{2} / k_{\mathrm{B}} T\right)$ \\
\hline \hline
\end{tabular}

\section{Standard deviation of the skyrmion diameters distribution}

The skyrmion average diameter is crucial to compute: (1) the standard deviation $\sigma_{\left\langle D_{\text {sky }}\right\rangle}$ of the diameter distribution expressing the deviation from the average skyrmion diameter $\left\langle D_{\text {sky }}\right\rangle$ and (2) the skyrmion configurational entropy $S$.

First, we calculate $\sigma_{\left\langle D_{\text {sky }}\right\rangle}=\left(C_{\text {sky }}^{a v} \pi \int_{0}^{\infty} d D_{\text {sky }}\left(D_{\text {sky }}-\right.\right.$ $\left.\left.\left\langle D_{\text {sky }}\right\rangle\right)^{2} D_{\text {sky }}^{2} e^{-\left(a / k_{\mathrm{B}} T\right)\left(D_{\text {sky }}-\left\langle D_{\text {sky }}\right\rangle\right)^{2}}\right)^{1 / 2}$ as an integral over the $\mathrm{MB}$ distribution centered at the average skyrmion diameter with $C_{\text {sky }}^{a v}=\left(2 \pi \int_{0}^{\infty} d D_{\text {sky }} D_{\text {sky }}^{2} e^{-\left(a / k_{B} T\right)\left(D_{\text {sky }}-\left\langle D_{\text {sky }}\right\rangle\right)^{2}}\right)^{-1}$ the normalization constant obtained after a normalization to unity of the MB distribution centered at the average diameter $\left\langle D_{\text {sky }}\right\rangle$ with $\left\langle D_{\text {sky }}\right\rangle=\left\langle D_{\text {sky }}(T)\right\rangle$ for every external field, obtaining

$$
\sigma_{\left\langle D_{\text {sky }}\right\rangle}(T)=\sqrt{\frac{k_{\mathrm{B}} T}{2 a}\left(\frac{3 k_{\mathrm{B}} T+2 a\left\langle D_{\text {sky }}\right\rangle^{2}}{k_{\mathrm{B}} T+2 a\left\langle D_{\text {sky }}\right\rangle^{2}}\right)},
$$

with $a=a(T)$.

In Fig. 4(b), we compare the standard deviation obtained from micromagnetic simulations and Eq. (4), observing that they match well in the region of energetic metastability.

\section{E. Configurational entropy}

We now outline the computation of the skyrmion configurational entropy that represents one of the key results of this study. We wish to remind that the source of this entropy is mainly due to the skyrmion internal deformations and thermal-breathing mode [35-37]. In this respect, very recently it has been shown that it is possible to get evidence for the configurational entropy in 2D skyrmionlike magnetic structures, defined in the framework of information theory, from the topological charge density [58].

To calculate the configurational entropy of a single skyrmion, we employ the definition of the Boltzmann order function $H_{0}$ for a dilute ideal gas [39], which represents a measure of order and is proportional to the MB distribution, which is the solution of the Boltzmann equation at thermodynamic equilibrium. Indeed, $H_{0}$ is a quantity defined as the opposite of the entropy $S$ at equilibrium, namely $H_{0}=$ $-S / k_{\mathrm{B}}$ [39], with $H_{0}<0$. According to this definition, $S$ gives the direct connection between the statistics of a microcanonical ensemble and thermodynamics. In the continuous case applied to our framework, where minor changes of the skyrmion size occur along the radial direction but also along the thickness, $H_{0}$ can be written as a $3 \mathrm{D}$ functional integral representing the statistical average $\left\langle\ln f_{0}\right\rangle$ over all spatial directions:

$$
H_{0}=2 \pi \int_{0}^{\infty} d D_{\text {sky }} D_{\text {sky }}^{2} f_{0} \ln f_{0}
$$

with $f_{0}=C_{\text {sky }} e^{-\left(a / k_{B} T\right)\left(D_{\text {sky }}-\left\langle D_{\text {sky }}\right\rangle\right)^{2}}$ the Gaussian distribution of the skyrmion diameters at thermodynamic equilibrium centered at the average skyrmion diameter, which has the meaning of a probability density in statistical mechanics.

The skyrmion configurational entropy turns out to be $\left(S=-k_{\mathrm{B}} H_{0}\right)$

$$
\begin{aligned}
S= & k_{\mathrm{B}}\left[\ln \left(\frac{\left(k_{\mathrm{B}} T\right)^{3 / 2}+2\left(k_{\mathrm{B}} T\right)^{1 / 2} a\left\langle D_{\text {sky }}\right\rangle^{2}}{a^{3 / 2}\left\langle D_{\text {sky }}\right\rangle^{2} t}\right)\right. \\
& \left.+\frac{1}{2}\left(\frac{3 k_{\mathrm{B}} T+2 a\left\langle D_{\text {sky }}\right\rangle^{2}}{k_{\mathrm{B}} T+2 a\left\langle D_{\text {sky }}\right\rangle^{2}}\right)\right]+S_{0},
\end{aligned}
$$

with $S_{0}=k_{\mathrm{B}}\left(\ln 4+\frac{1}{2} \ln \pi\right)$ a constant [see Appendix A for the details of the calculations leading to Eq. (6)].

As expected, the configurational entropy has a geometric, thermal, and magnetic parameters dependence. $S$ depends on the size of the skyrmion via $\left\langle D_{\text {sky }}\right\rangle$, confirming the link with the thermal-breathing mode [Figs. 1(b)-1(e)], decreases with decreasing temperature, denoting the strict connection with temperature effects until a minimum temperature close to $1 \mathrm{~K}$ (see Sec. III F for more details), as well as depending on the magnetic parameters via the coefficient $a$. Note that Eq. (6) is a general result since it is independent of the chosen skyrmion distribution texture. Indeed, different skyrmion distribution texture would lead to energy profiles again reproducible in the neighborhood of the minimum by means of a parabolic dependence.

However, as $T \rightarrow 0 \mathrm{~K}$, we get, from Eq. (6), $S \rightarrow-\infty$ apparently contradicting Nernst's theorem or third principle of thermodynamics according to which entropy of a crystalline body equals zero at absolute zero temperature [see also the discussion of Eq. (9)]. This result is not surprising and agrees with the well-known one of the Sackur-Tetrode entropy equation for an ideal gas [38]. Indeed, in both cases the derivation is classical, resulting from the application of the classical MB statistics.

Figure 5(a) shows the skyrmion configurational entropy, calculated according to Eq. (6), as a function of temperature for three different external fields $(0,25$, and $50 \mathrm{mT})$. The entropy increases with increasing temperature and its increase is more remarkable in the absence of an external bias field. This behavior reflects the higher disorder due to the larger deformations and thermal-breathing mode of the skyrmion at room temperature [35]. This disorder is partially reduced by the ordering effect of the external bias field.

Figure 5(b) illustrates the trend of the configurational entropy as a function of the external field at fixed temperature $(T=300 \mathrm{~K})$. It is evident the entropy decreases due to the 

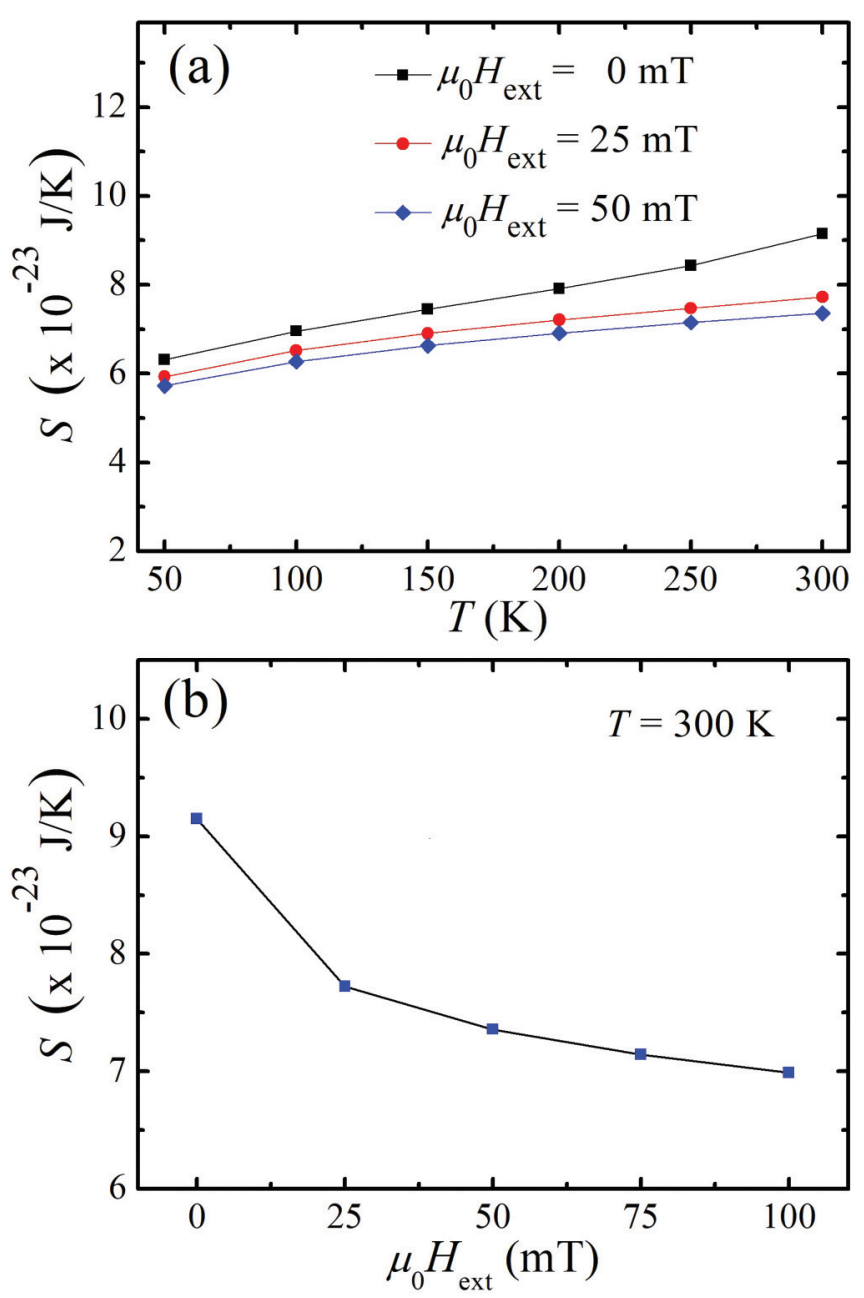

FIG. 5. Configurational entropy analytically calculated from Eq. (6) as a function of (a) temperature for $\mu_{0} H_{\mathrm{ext}}=0,25$, and $50 \mathrm{mT}$, as indicated in the legend, and of (b) the external field at $T=300 \mathrm{~K}$

external bias field that leads to a reduction of the disorder of the system and this trend occurs also at other temperatures.

\section{F. Behavior of $\left\langle D_{\text {sky }}\right\rangle, \sigma_{D \text { sky }}$, and $S$ at low temperature}

It is interesting to derive the low-temperature behavior of the average skyrmion diameter. We assume, for the whole range of temperatures studied $(0-300 \mathrm{~K}) a(T)=a_{0}+c T$ [see Fig. 2(a)], with $a_{0}=a(T=0 \mathrm{~K})$ and $c<0$ a coefficient expressed in $\mathrm{J} /\left(\mathrm{m}^{2} \mathrm{~K}\right)$, and, at low temperatures, $D_{0 \text { sky }}(T)=$ $D_{0 \text { sky }}(T=0 \mathrm{~K})+d T$, with $d$ a coefficient expressed in $\mathrm{m} / \mathrm{K}$.

For $T \rightarrow 0 \mathrm{~K}$, we get, from a numerical calculation, that $\left\langle D_{\text {sky }}(T \rightarrow 0 \mathrm{~K})\right\rangle=D_{0 \text { sky }}(T=0 \mathrm{~K})$. At low temperature, the expansion of Eq. (3) to first order, via $\left\langle D_{\text {sky }}(T \rightarrow 0 \mathrm{~K})\right\rangle=$ $D_{0 \text { sky }}(T=0 \mathrm{~K})$, yields

$$
\left\langle D_{\text {sky }}(T)\right\rangle_{T \rightarrow 0 \mathrm{~K}} \approx D_{0 \text { sky }}^{0}+\frac{k_{\mathrm{B}}}{a_{0} D_{0 \text { sky }}^{0}} T
$$

with $a_{0}=a(T=0 \mathrm{~K})$ and $D_{0 \text { sky }}^{0}=D_{0 \text { sky }}(T=0 \mathrm{~K})$.
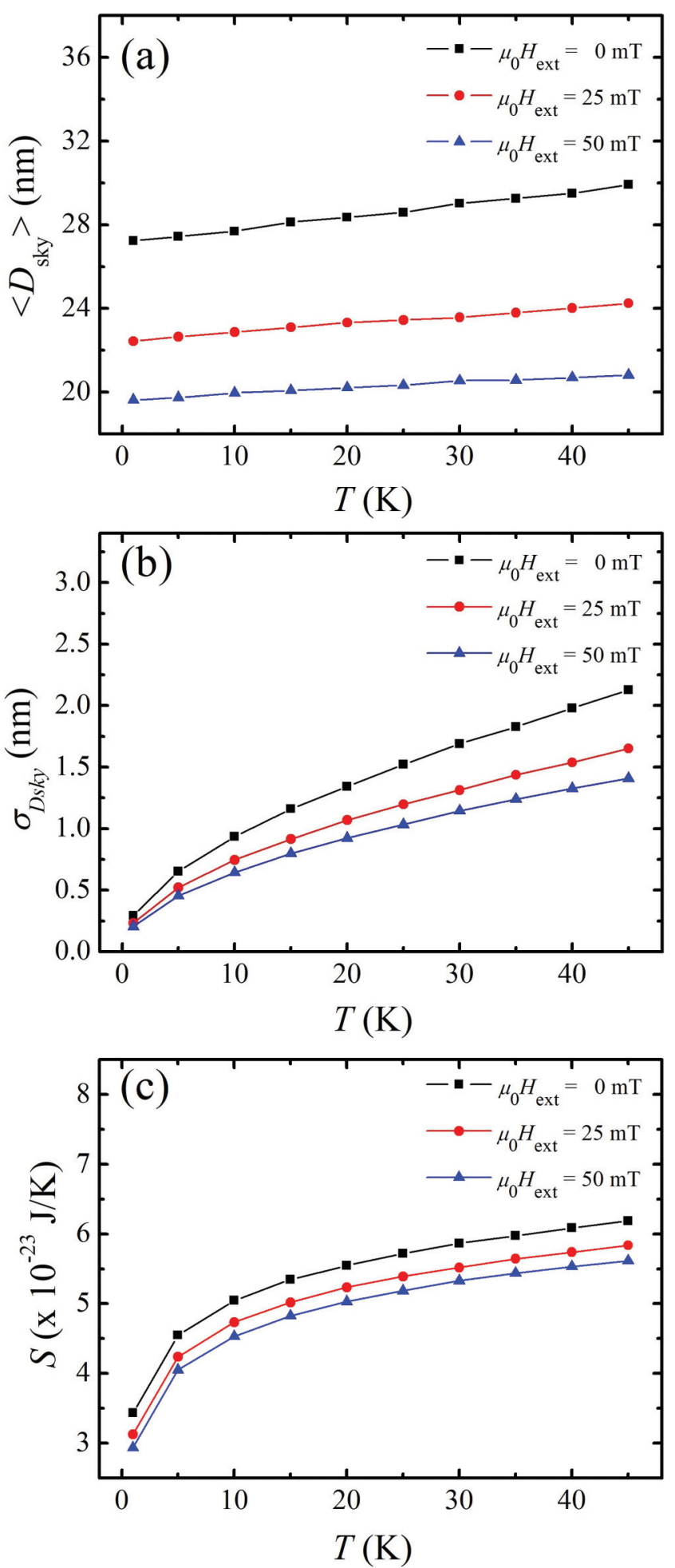

FIG. 6. Low-temperature behavior of (a) average skyrmion diameter, as obtained by Eq. (3), (b) standard deviation of the skyrmion diameters distribution, as obtained by Eq. (4), and (c) configurational entropy, as obtained by Eq. (6).

Equation (7) expresses, in the regime of low temperatures, a linear dependence of the average skyrmion diameter on temperature, as it can be observed in Fig. 6(a) for different combinations of low temperature and external bias field. 
At low temperatures $(T \rightarrow 0 \mathrm{~K})$, via a series expansion to the first order of Eq. (4) and taking into account the abovementioned assumptions on $a$ and $D_{0 \text { sky }}$, the standard deviation turns out to be

$$
\sigma_{\left\langle D_{\text {sky }}\right\rangle(T \rightarrow 0 \mathrm{~K})} \approx \sqrt{\frac{k_{\mathrm{B}} T}{2 a_{0}}} .
$$

Hence, at low temperatures the standard deviation has a square-root proportionality on temperature, as shown in Fig. 6(b).

In the same way, from Eq. (6) the configurational entropy expanded to the first order in $T$ for $T \rightarrow 0 \mathrm{~K}$ reads

$$
\begin{aligned}
& S_{(T \rightarrow 0 \mathrm{~K})} \\
& \quad \approx k_{\mathrm{B}}\left[\ln \left(\frac{1}{t}\left(\frac{k_{\mathrm{B}} T}{a_{0}}\right)^{1 / 2}\right)+\left(\frac{k_{\mathrm{B}}}{a_{0}\left(D_{0 \text { sky }}^{0}\right)^{2}}-\frac{c}{2 a_{0}}\right) T\right]+S_{1},
\end{aligned}
$$

with $\quad D_{0 \text { sky }}^{0}=\left\langle D_{\text {sky }}(T=0 \mathrm{~K})\right\rangle \quad$ and $\quad S_{1}=k_{B}(3 \ln 2+$ $1 / 2 \ln \pi+1 / 2)$.

The first term of the second member has a logarithmic dependence on $T$, hence giving the divergence of $S$ for $T=$ $0 \mathrm{~K}$. On the other hand, the second term expresses the linear dependence of $S$ on $T$. This latter term receives contribution from both the logarithmic and the fractional terms of the second member of Eq. (6) when expanded to the first order in $T$.

Figure 6(c) shows $S$ vs $T$ for $1<T<50 \mathrm{~K}$. It is evident that the deviation from the linear behavior due to the effect of $\ln (T)$ at low temperature causes a sudden drop of $S$. For $0<T<1 \mathrm{~K}$ the model is not anymore valid $(S \rightarrow-\infty$ for $T \rightarrow 0 \mathrm{~K}$ ) and the amplitude of this interval depends on the magnetic and geometric parameters used in the calculations. Equations (7)-(9) show a good agreement with Eqs. (3), (4), and (6), respectively, up to $10 \mathrm{~K}$ (not shown).

\section{G. Energy fluctuations}

The skyrmion configurational entropy is crucial to determine the skyrmion fluctuations of energy around the average energy $\langle E\rangle$. In principle, the skyrmion diameters distribution is supposed to be in contact with a heat reservoir forming a canonical ensemble, and the average energy is determined by the temperature of the heat reservoir itself. However, due to the high number of degrees of freedom represented by the skyrmion diameters population, we can suppose that the fluctuations of energy are small, treating the canonical ensemble in the same way as a microcanonical ensemble. This hypothesis is confirmed by the calculation of the energy fluctuations that are small if compared to the average energy as shown above.

The evaluation of the mean-square fluctuation of the skyrmion energy is based on the calculation of the partition function in the discrete limit of a microcanonical ensemble yielding $\left\langle\delta E^{2}\right\rangle \simeq k_{\mathrm{B}} T^{3}\left(2 \frac{\partial S}{\partial T}+T \frac{\partial^{2} S}{\partial T^{2}}-\frac{\partial^{2}\langle E\rangle}{\partial T^{2}}\right)$, with $\left\langle\delta E^{2}\right\rangle=$ $\left\langle E^{2}\right\rangle-\langle E\rangle^{2}$ and $\langle E\rangle \approx a\left\langle D_{\text {sky }}{ }^{2}\right\rangle$ the average energy (see Appendix $\mathrm{B}$ for details about its calculation). The average energy takes into account the contribution strictly depending on skyrmion diameter but not the one resulting from $b=$


FIG. 7. (a) Mean-square fluctuation of the energy of the average skyrmion energy as a function of temperature for $\mu_{0} H_{\text {ext }}=0,25$, and $50 \mathrm{mT}$. (b) As in (a) but for the fractional mean-square fluctuation of the energy.

$b(T)$ that determines only a shift of the energy minimum of about $10^{-17} \mathrm{~J}$ weakly dependent on temperature. The key result is that skyrmion mean-square fluctuation of the energy is apportioned between an entropic and an average energy dependence. We have found that there is a dependence of the form $\left\langle\delta E^{2}\right\rangle \propto\left(k_{\mathrm{B}} T\right)^{2}$ (see Appendix B for the details) and the constant of proportionality is not simply $3 / 2$ like for the 3D MB ideal-gas distribution but depends on magnetic and geometric parameters.

In Fig. 7(a), we display the mean-square fluctuation of the energy of the skyrmion diameters distribution as a function of temperature determined via a calculation of the entropy and the average energy derivatives with respect to $T .\left\langle\delta E^{2}\right\rangle$ shows a quadratic increase with increasing temperature with a much lower curvature for $\mu_{0} H_{\mathrm{ext}}=25$ and $50 \mathrm{mT}$. In particular, for $\mu_{0} H_{\text {ext }}=0 \mathrm{mT},\left\langle\delta E^{2}\right\rangle$ receives contributions from both the entropy and the average energy resulting in about $10^{-41} \mathrm{~J}^{2}$, while, for nonvanishing external bias field, the average energy 
contribution is negligible due to the almost linear behavior of $\langle E\rangle$ vs $T$ so that $\left\langle\delta E^{2}\right\rangle \approx 10^{-42} \mathrm{~J}^{2}$.

The quadratic increase of $\left\langle\delta E^{2}\right\rangle$ vs $T$ is analogous to that of the mean-square fluctuation of the energy of gas particles. However, note that the external bias field reduces $\left\langle\delta E^{2}\right\rangle$ on average of one order of magnitude. The calculated mean-square fluctuation of the energy is about one order of magnitude smaller than the one calculated according to the well-known statistical relation $\left\langle\delta E^{2}\right\rangle=k_{\mathrm{B}} T^{2} \frac{\partial\langle E\rangle}{\partial T}$. This slight discrepancy is not surprising because those fluctuations are calculated using the partition function in the continuous limit of a canonical ensemble [39].

To establish whether the skyrmion fluctuations of energy are relevant, we compare their order of magnitude with that of the average energy calculating the fractional mean-square fluctuation of the energy: $\left\langle\delta E^{2}\right\rangle /\langle E\rangle^{2} \propto\left(k_{\mathrm{B}} T\right)^{2} /\langle E\rangle^{2}$. For the system studied and the range of temperatures considered, on average for $\mu_{0} H_{\text {ext }}=0 \mathrm{mT}\left\langle\delta E^{2}\right\rangle \approx 10^{-41} \mathrm{~J}^{2}$ [see Fig. 7(b)]. As $\langle E\rangle^{2} \approx 10^{-39} \mathrm{~J}^{2}$ we get $\frac{\left\langle\delta E^{2}\right\rangle}{\langle E\rangle^{2}} \simeq 10^{-2}$, resulting in fluctuation of the energy that is about $10 \%$ of the average energy. Looking at Fig. 7(b), the fractional mean-square fluctuation of the energy does depend on $T$ and this dependence is accentuated at higher temperatures. This is not surprising because, unlike ideal gas, there is not only an explicit dependence on $T$ of the average energy but also via $a=a(T)$ and $\left\langle D_{\text {sky }}\right\rangle=\left\langle D_{\text {sky }}(T)\right\rangle$ in turn depending on the scaled magnetic parameters. In the presence of an external bias field, the average energy increases with increasing $H_{\text {ext }}$ contributing to the reduction of the fractional mean-square fluctuation of the energy $\left(\frac{\left\langle\delta E^{2}\right\rangle}{\langle E\rangle^{2}} \simeq 10^{-3}\right)$ that still has a dependence on $T$ even though much less accentuated with respect to the case for $\mu_{0} H_{\text {ext }}=0 \mathrm{mT}$. This corresponds to fluctuation of the energy that is about $3 \%$ of the average energy. In principle, the energy fluctuations albeit small could be detected via calorimetric measurements enabling the determination of the skyrmion entropy. The quantitative estimate of energy fluctuations confirms the initial assumption of studying skyrmion diameters population in a microcanonical ensemble that is strengthened when an external bias field is applied to the system.

\section{CONCLUSIONS}

In summary, we have shown that it is possible to describe the statistical behavior of the skyrmion diameter in the presence of thermal fluctuations by using a statistical thermodynamic analogy with the noninteracting molecules of an ideal diluted gas. This analogy is valid in the region of energetic metastability for the skyrmion where the following hypotheses are verified: (i) the skyrmion energy close to the minimum exhibits a parabolic profile and (ii) the skyrmion diameters population follows a MB statistical distribution. We confirmed those hypotheses with the results of micromagnetic simulations. Therefore, we have developed an analytical model able to describe the statistical behavior of skyrmion diameter of the magnetic (average value, standard deviation, and distribution) as well as to calculate its configurational entropy linked to the thermal-breathing mode and internal deformations. In the low-temperature limit, the average skyrmion diameter has a linear dependence on $T$, while the configurational entropy shows a logarithmic behavior and a dip at very low temperatures leading to a deviation from the linear behavior. From the calculation of the partition function of a microcanonical ensemble, we have expressed the energy fluctuations both as a function of the entropy and of the average energy of the magnetic skyrmion. Those results can be used to study phase transitions involving magnetic skyrmions and their relaxation properties.

\section{ACKNOWLEDGMENTS}

R.T. and G.F. acknowledge the project "ThunderSKY" funded by the Hellenic Foundation for Research and Innovation and the General Secretariat for Research and Technology, under Grant No. 871. R.Z. acknowledges support by Gruppo Nazionale per la Fisica Matematica (GNFM) and Istituto Nazionale di Alta Matematica (INdAM) "F. Severi." O.C.-F. also acknowledges the Spanish Ministry of Economy and Competitiveness under Project No. FIS201678591-C3-3-R and the support from the Messina University with its Visiting Research program. G. F., O.C.-F., and M.C. would like to acknowledge networking support by the COST Action No. CA17123 "Ultrafast opto magneto electronics for non-dissipative information technology." R.Z. thanks Daniele Pinna for fruitful discussions.

\section{APPENDIX A: CALCULATION OF THE CONFIGURATIONAL ENTROPY}

We recall Eq. (5) of the main text expressing the 3D Boltzmann-order function at thermodynamic equilibrium:

$$
H_{0}=2 \pi \int_{0}^{\infty} d D_{\text {sky }} D_{\text {sky }}^{2} f_{0} \ln f_{0},
$$

with $2 \pi$ resulting from the integration over the polar and azimuthal angles. The computation of the integral via $f_{0}=$ $C_{\text {sky }}^{\text {av }} e^{-\left(a / k_{B} T\right)\left(D_{\text {sky }}-\left\langle D_{\text {sky }}\right\rangle\right)^{2}}$ and the substitution $C_{\text {sky }}^{\text {av }} \rightarrow C_{\text {sky }}^{\text {av }}\langle V\rangle$ to make the argument of the logarithmic function dimensionless yields

$$
\begin{aligned}
H_{0}= & C_{\text {sky }}^{\mathrm{av}}\left(\frac{\pi}{a}\right)^{3 / 2}\left(k_{\mathrm{B}} T\right)^{1 / 2}\left[\left(k_{\mathrm{B}} T+2 a\left\langle D_{\text {sky }}\right\rangle^{2}\right) \ln \left(C_{\text {sky }}^{\mathrm{av}}\langle V\rangle\right)\right. \\
& \left.-\frac{1}{2}\left(3 k_{\mathrm{B}} T+2 a\left\langle D_{\text {sky }}\right\rangle^{2}\right)\right] .
\end{aligned}
$$

Here, $\langle V\rangle$ is the average skyrmion volume that can be approximately expressed in terms of the skyrmion diameter as $\langle V\rangle \simeq 1 / 4 \quad \pi\left\langle D_{\text {sky }}\right\rangle^{2} t$ taking into account that $\left\langle D_{\text {sky }}^{2}\right\rangle \approx\left\langle D_{\text {sky }}\right\rangle^{2}$ (note however that $\left.\left\langle R_{\text {sky }}\right\rangle \neq 1 /{ }_{2}\left\langle D_{\text {sky }}\right\rangle\right)$. We get the constant $C_{\text {sky }}^{\text {av }}$ via the normalization condition of the MB distribution to unity, $C_{\text {sky }}^{\text {av }}=\frac{1}{2 \pi}\left[\int_{0}^{\infty} d D_{\text {sky }} D_{\text {sky }}^{2} e^{-\left(a / k_{B} T\right)\left(D_{\text {sky }}-\left\langle D_{\text {sky }}\right\rangle\right)^{2}}\right]^{-1}, \quad$ resulting in $C_{\mathrm{sky}}^{\mathrm{av}}=\left(\frac{a}{\pi}\right)^{3 / 2} \frac{1}{\left(k_{\mathrm{B}} T\right)^{1 / 2}}\left(\frac{1}{k_{\mathrm{B}} T+2 a\left\langle D_{\mathrm{sky}}\right)^{2}}\right)$. Substituting $C_{\mathrm{sky}}^{\mathrm{av}}$ and $\langle V\rangle$ into $H_{0}$, we get

$$
\begin{aligned}
H_{0} \simeq & \ln \left[\frac{(a)^{3 / 2}\left\langle D_{\text {sky }}\right\rangle^{2} t}{\left(k_{\mathrm{B}} T\right)^{3 / 2}+2\left(k_{\mathrm{B}} T\right)^{1 / 2} a\left\langle D_{\text {sky }}\right\rangle^{2}}\right] \\
& -\frac{1}{2}\left(\frac{3 k_{\mathrm{B}} T+2 a\left\langle D_{\text {sky }}\right\rangle^{2}}{k_{\mathrm{B}} T+2 a\left\langle D_{\text {sky }}\right\rangle^{2}}\right)-\ln 4-\frac{1}{2} \ln \pi,
\end{aligned}
$$

with $H_{0}<0$. 
Skyrmion entropy at the thermodynamic equilibrium is calculated as $S=-k_{\mathrm{B}} H_{0}$ after substituting $C$ and $\langle V\rangle$ :

$$
\begin{aligned}
S \simeq k_{\mathrm{B}} & {\left[\ln \left(\frac{\left(k_{\mathrm{B}} T\right)^{3 / 2}+2\left(k_{\mathrm{B}} T\right)^{1 / 2} a\left\langle D_{\text {sky }}\right\rangle^{2}}{a^{3 / 2}\left\langle D_{\text {sky }}\right\rangle^{2} t}\right)\right.} \\
& \left.+\frac{1}{2}\left(\frac{3 k_{\mathrm{B}} T+2 a\left\langle D_{\text {sky }}\right\rangle^{2}}{k_{\mathrm{B}} T+2 a\left\langle D_{\text {sky }}\right\rangle^{2}}\right)\right]+S_{0},
\end{aligned}
$$

with $\quad a=a(T), \quad\left\langle D_{\text {sky }}\right\rangle=\left\langle D_{\text {sky }}(T)\right\rangle, \quad$ and $\quad S_{0}=$ $k_{\mathrm{B}}\left(\ln 4+\frac{1}{2} \ln \pi\right)$. Equation (A4) is Eq. (6) of the main text.

\section{APPENDIX B: CALCULATION OF THE MAGNETIZATION DISTRIBUTION AND OF THE ENERGY FLUCTUATIONS}

The skyrmion entropy is crucial to determine: (1) the magnetization distribution as a function of temperature at fixed external bias field and (2) the skyrmion fluctuations of energy around the average energy $<E>$.

The calculation of the magnetization distribution is based on the hyperbolic law expressing the average skyrmion diameter as a function of $B=\mu_{0} H_{\text {ext }}$ at fixed $T$ as observed by Romming et al. for $T=4.2 \mathrm{~K}$ [59] and confirmed by our model at higher temperatures:

$$
\left\langle D_{\text {sky }}(B)\right\rangle=\frac{B_{0}}{B+B_{0}}\left\langle D_{\text {sky }}^{0}\right\rangle
$$

with $B$ expressed here as $J\left(B \rightarrow \mu_{0} H_{\text {ext }} M_{\mathrm{s}} V\right), B_{0}$ a parameter expressed in $J$ dependent on $T, B_{0}=B_{0}(T)$ that can be obtained from a fit to experimental data (measured skyrmion diameters) for $B=0 \mathrm{~J}$ [59] and $\left\langle D_{\text {sky }}^{0}\right\rangle=\left\langle D_{\text {sky }}(B=0 \mathrm{~J})\right\rangle$ with $\left\langle D_{\text {sky }}^{0}\right\rangle=\left\langle D_{\text {sky }}^{0}(T)\right\rangle$.

First, we calculate the entropy dependence on the bias external field at fixed temperature. Substituting Eq. (B1) into Eq. (6) we get the skyrmion entropy dependence on $B$ at fixed $T$ :

$$
S(B) \simeq k_{\mathrm{B}}\left[\left(\ln \left[\frac{\left(\left(k_{\mathrm{B}} T\right)^{3 / 2}+2\left(k_{\mathrm{B}} T\right)^{1 / 2} a\left(\frac{B_{0}}{B+B_{0}}\left\langle D_{\text {sky }}^{0}\right\rangle\right)^{2}\right)}{(a)^{3 / 2}\left(\frac{B_{0}}{B+B_{0}}\left\langle D_{\text {sky }}^{0}\right\rangle\right)^{2} t}\right]\right)+\frac{1}{2}\left(\frac{3 k_{\mathrm{B}} T+2 a\left(\frac{B_{0}}{B+B_{0}}\left\langle D_{\text {sky }}^{0}\right\rangle\right)^{2}}{k_{\mathrm{B}} T+2 a\left(\frac{B_{0}}{B+B_{0}}\left\langle D_{\text {sky }}^{0}\right\rangle\right)^{2}}\right)\right]+S_{0} .
$$

We immediately find, using Maxwell's relation $\left(\frac{\partial S}{\partial B}\right)_{T}=\left(\frac{\partial m}{\partial T}\right)_{B}$ with $m$ the normalized magnetization after multiplying by $M_{\mathrm{s}}$ :

$$
\left(\frac{\partial M}{\partial T}\right)_{\mathrm{B}}=2 M_{\mathrm{s}}\left[k_{\mathrm{B}}^{2} T\left(\frac{\left(B+B_{0}\right)\left(k_{\mathrm{B}} T\left(B+B_{0}\right)^{2}+4 a\left\langle D_{\mathrm{sky}}^{0}\right)^{2} B_{0}^{2}\right)}{\left(k_{\mathrm{B}} T\left(B+B_{0}\right)^{2}+2 a\left(D_{\mathrm{sky}}^{0}\right)^{2} B_{0}^{2}\right)^{2}}\right)\right],
$$

with $a=a(T)$ and $M_{\mathrm{s}}=M_{\mathrm{s}}(T=0 \mathrm{~K})$. For $B=0 \mathrm{~J}$ $\left(\mu_{0} H_{\mathrm{ext}}=0 \mathrm{mT}\right)$, the magnetization distribution as a function of temperature at different $T$ is

$$
\left(\frac{\partial M}{\partial T}\right)_{B=0 \mathrm{~J}}=2 \frac{M_{\mathrm{s}}}{B_{0}}\left[k_{\mathrm{B}}^{2} T\left(\frac{k_{\mathrm{B}} T+4 a\left\langle D_{\text {sky }}^{0}\right)^{2}}{\left(k_{\mathrm{B}} T+2 a\left(D_{\mathrm{sky}}^{0}\right)^{2}\right)^{2}}\right)\right] .
$$

The magnetization distribution in the ferromagnetic state depends on $B_{0}$ and on the relevant parameters characterizing skyrmion energy, $a$ and $\left\langle D_{\text {sky }}^{0}\right\rangle$ all functions of $T$. From Eq. (B4) the trend of $B_{0}$ (in J) could be fit as a function of $T$ that makes $\frac{\partial M}{\partial T}=$ cost as predicted by the atomistic model [35] according to which the saturation magnetization decreases linearly with increasing $T$ for the range of temperatures studied.

We now outline the calculation of point (2) above, the skyrmion fluctuations of energy around the average value $\langle E\rangle$. Let us start from the statistical thermodynamic relation linking the average energy with the partition function $Z,\langle E\rangle=$ $-\frac{\partial \ln Z}{\partial \beta}$, with $\beta=1 / k_{\mathrm{B}} T$. This implies that $\langle E\rangle^{2}=\left(\frac{\partial \ln Z}{\partial \beta}\right)^{2}$.

Owing to our first assumption on the parabolic fit, the average skyrmion energy can be approximately written as $\langle E\rangle \approx a\left\langle D_{\text {sky }}^{2}\right\rangle$, where $\left\langle D_{\text {sky }}^{2}\right\rangle=\left\langle D_{\text {sky }}^{2}(T)\right\rangle$ is the average of the square of the skyrmion diameter over the MB distribution of skyrmion diameters $\left\langle D_{\text {sky }}^{2}(T)\right\rangle=$ $C_{\text {sky } 0} 2 \pi \int_{0}^{\infty} d D_{\text {sky }} D_{\text {sky }}^{3} e^{-\left(a / k_{B} T\right)\left(D_{\text {sky }}-D_{0 \text { sky }}\right)^{2}}$, with $C_{\text {sky } 0}=$ $\left(2 \pi \int_{0}^{\infty} d D_{\text {sky }} D_{\text {sky }}^{2} e^{-\left(a / k_{B} T\right)\left(D_{\text {sky }}-D_{0 \text { sky }}\right)^{2}}\right)^{-1}$. The calculation of the integral yields

$$
\langle E\rangle \simeq \frac{5 k_{\mathrm{B}} T+2 a\left(D_{0} \text { sky }\right)^{2}}{2},
$$

with an explicit dependence on $T$ but also an implicit dependence on $T$ via $a=a(T)$ and $D_{0 \text { sky }}=D_{0 \text { sky }}(T)$. In the calculation of $\langle E\rangle$ we have taken into account the contribution of the skyrmion energy strictly depending on the skyrmion diameter since $b=b(T)$ accounts only for the translation of the energy minimum. Note that the average energy in Eq. (B5) reduces to $\langle E\rangle \simeq \frac{5 k_{\mathrm{B}} T}{2}$ setting $D_{0 \text { sky }}=0 \mathrm{~nm}$. The well-known result $\langle E\rangle \simeq \frac{3}{2} k_{\mathrm{B}} T$ for the average energy per particle of an ideal gas would be recovered considering $\langle E\rangle \simeq$ $a\left\langle\left(D_{\text {sky }}-D_{0 \text { sky }}\right)^{2}\right\rangle$. However, the skyrmion average energy written in this form would be proportional to the average square deviation of the skyrmion diameter from the equilibrium diameter and numerically is one order of magnitude smaller than the one expressed in Eq. (B5).

To get an explicit expression of the fluctuations of energy, we write the partition function of the system that can be regarded as a controlling function able to determine the average energy of the system itself. In the continuum limit, we write the partition function of a canonical ensemble as an integral over Boltzmann factors $e^{-\beta E(x)}, Z=C_{N} \int e^{-\beta E(x)} d x$ with $C_{N}$ a normalization constant. We now express the average of the square of the energy as a function of the partition function. We write the average of the square of the energy in the continuous 
limit in a general form as

$$
\left\langle E^{2}\right\rangle=\frac{C}{Z} \int[E(x)]^{2} e^{-\beta E(x)} d x,
$$

where $C$ is a normalization constant and $E(x)$ is the energy depending on the generic continuous variable $x$ that in our case corresponds to the skyrmion diameter. We cast Eq. (B6) in the form $\left\langle E^{2}\right\rangle=\frac{C}{Z} \int \frac{\partial^{2}}{\partial \beta^{2}}\left(e^{-\beta E(x)}\right) d x=\frac{1}{Z} \frac{\partial^{2} Z}{\partial \beta^{2}}=\frac{\partial^{2} \ln Z}{\partial \beta^{2}}+$ $\left[\frac{1}{Z}\left(\frac{\partial Z}{\partial \beta}\right)\right]^{2}=\frac{\partial^{2} \ln Z}{\partial \beta^{2}}+\left(\frac{\partial \ln Z}{\partial \beta}\right)^{2}$. Hence, we get $\left\langle E^{2}\right\rangle-\langle E\rangle^{2}=$ $\frac{\partial^{2} \ln Z}{\partial \beta^{2}}+\left(\frac{\partial \ln Z}{\partial \beta}\right)^{2}-\left(\frac{\partial \ln Z}{\partial \beta}\right)^{2}=\frac{\partial^{2} \ln Z}{\partial \beta^{2}}$. The square fluctuation of the energy expressed as a function of $T$ takes the form

$$
\left\langle\delta E^{2}\right\rangle=k_{\mathrm{B}}^{2} T^{4} \frac{\partial^{2} \ln Z}{\partial T^{2}},
$$

being $\left\langle\delta E^{2}\right\rangle=\left\langle E^{2}\right\rangle-\langle E\rangle^{2}$.

The partition function of the statistical ensemble of skyrmion diameters under study that we have regarded as a microcanonical ensemble can be approximately expressed as $Z \simeq W e^{-\left(\langle E\rangle / k_{\mathrm{B}} T\right)}$, where $W$ is the statistical multiplicity of the energy level having value $\langle E\rangle$ because of two approximations. The first approximation consists of treating a continuous variable as a discrete variable writing the partition function as a sum of states, each one characterized by a given energy. The second approximation consists of assuming, within the microcanonical ensemble, that all energies in the sum of states are equal to the average energy of the ensemble considering small energy fluctuations. Thanks to the above approximations, we are able to express the mean-square fluctuations of energy of Eq. (B7) via a combined dependence on the entropy and on the average energy of the magnetic skyrmion. Indeed, writing the partition function $Z$ of a microcanonical ensemble allows for determining, via the microcanonical expression $S=k_{\mathrm{B}} \ln W$, the simple relation linking the partition function of a microcanonical ensemble, the entropy, and the average energy [60]:

$$
\ln Z \simeq \frac{S}{k_{\mathrm{B}}}-\frac{\langle E\rangle}{k_{\mathrm{B}} T},
$$

starting from which $\left\langle\delta E^{2}\right\rangle$ can be easily calculated. Taking into account Eqs. (B7) and (B8) yields

$$
\left\langle\delta E^{2}\right\rangle \simeq k_{\mathrm{B}} T^{3}\left(2 \frac{\partial S}{\partial T}+T \frac{\partial^{2} S}{\partial T^{2}}-\frac{\partial^{2}\langle E\rangle}{\partial T^{2}}\right) .
$$

We note that mean-square fluctuations of energy around the mean energy of the skyrmion diameters population is apportioned between an entropy contribution and an average energy contribution. Equation (B9) is slightly different from the well-known expression of mean-square fluctuation of the energy in a canonical ensemble that depends only on the average energy.

$\left\langle\delta E^{2}\right\rangle$ has been determined via the numerical calculation of the derivatives with respect to $T$ of entropy and of the average energy of the magnetic skyrmion appearing in Eq. (B9) and turns out to be proportional to the square of the temperature, $\left\langle\delta E^{2}\right\rangle \propto\left(k_{\mathrm{B}} T\right)^{2}$ (as occurs for fluctuations of energy in an ideal gas) with a constant of proportionality that reduces with increasing the amplitude of the applied bias field.
[1] G. Finocchio, F. Büttner, R. Tomasello, M. Carpentieri, and M. Kläui, J. Phys. D: Appl. Phys. 49, 423001 (2016).

[2] W. Jiang, W. Zhang, G. Yu, M. B. Jungfleisch, P. Upadhyaya, H. Somaily, J. E. Pearson, Y. Tserkovnyak, K. L. Wang, O. Heinonen, S. G. E. te Velthuis, and A. Hoffmann, AIP Adv. 6, 055602 (2016).

[3] A. Soumyanarayanan, N. Reyren, A. Fert, and C. Panagopoulos, Nature (London) 539, 509 (2016).

[4] A. Fert, N. Reyren, and V. Cros, Nat. Rev. Mater. 2, 17031 (2017).

[5] I. Dzyaloshinsky, J. Phys. Chem. Solids 4, 241 (1958).

[6] T. Moriya, Phys. Rev. Lett. 4, 228 (1960).

[7] U. K. Rößler, A. N. Bogdanov, and C. Pfleiderer, Nature (London) 442, 797 (2006).

[8] S. Muhlbauer, B. Binz, F. Jonietz, C. Pfleiderer, A. Rosch, A. Neubauer, R. Georgii, and P. Boni, Science 323, 915 (2009).

[9] A. Neubauer, C. Pfleiderer, B. Binz, A. Rosch, R. Ritz, P. G. Niklowitz, and P. Böni, Phys. Rev. Lett. 102, 186602 (2009).

[10] C. Moreau-Luchaire, C. Moutafis, N. Reyren, J. Sampaio, C. A. F. Vaz, N. Van Horne, K. Bouzehouane, K. Garcia, C. Deranlot, P. Warnicke, P. Wohlhüter, J.-M. George, M. Weigand, J. Raabe, V. Cros, and A. Fert, Nat. Nanotechnol. 11, 444 (2016).

[11] O. Boulle, J. Vogel, H. Yang, S. Pizzini, D. de Souza Chaves, A. Locatelli, T. O. M. A. Sala, L. D. Buda-Prejbeanu, O. Klein, M. Belmeguenai, Y. Roussigné, A. Stashkevich, S. M. Chérif,
L. Aballe, M. Foerster, M. Chshiev, S. Auffret, I. M. Miron, and G. Gaudin, Nat. Nanotechnol. 11, 449 (2016).

[12] S. Woo, K. Litzius, B. Krüger, M.-Y. Im, L. Caretta, K. Richter, M. Mann, A. Krone, R. M. Reeve, M. Weigand, P. Agrawal, I. Lemesh, M.-A. Mawass, P. Fischer, M. Kläui, and G. S. D. Beach, Nat. Mater. 15, 501 (2016).

[13] A. Soumyanarayanan, M. Raju, A. L. Gonzalez Oyarce, A. K. C. Tan, M.-Y. Im, A. P. Petrović, P. Ho, K. H. Khoo, M. Tran, C. K. Gan, F. Ernult, and C. Panagopoulos, Nat. Mater. 16, 898 (2017).

[14] A. K. Nayak, V. Kumar, T. Ma, P. Werner, E. Pippel, R. Sahoo, F. Damay, U. K. Rößler, C. Felser, and S. S. P. Parkin, Nature (London) 548, 561 (2017).

[15] M. Hoffmann, B. Zimmermann, G. P. Müller, D. Schürhoff, N. S. Kiselev, C. Melcher, and S. Blügel, Nat. Commun. 8, 308 (2017).

[16] F. Büttner, I. Lemesh, M. Schneider, B. Pfau, C. M. Günther, P. Hessing, J. Geilhufe, L. Caretta, D. Engel, B. Krüger, J. Viefhaus, S. Eisebitt, and G. S. D. Beach, Nat. Nanotechnol. 12, 1040 (2017).

[17] K. Everschor-Sitte, M. Sitte, T. Valet, A. Abanov, and J. Sinova, New J. Phys. 19, 092001 (2017).

[18] J. Zázvorka, F. Jakobs, D. Heinze, N. Keil, S. Kromin, S. Jaiswal, K. Litzius, G. Jakob, P. Virnau, D. Pinna, K. Everschor-Sitte, A. Donges, U. Nowak, and M. Kläui, Nat. Nanotechnol. (2019). 
[19] W. Jiang, P. Upadhyaya, W. Zhang, G. Yu, M. B. Jungfleisch, F. Y. Fradin, J. E. Pearson, Y. Tserkovnyak, K. L. Wang, O. Heinonen, S. G. E. te Velthuis, and A. Hoffmann, Science 349, 283 (2015).

[20] W. Jiang, X. Zhang, G. Yu, W. Zhang, X. Wang, M. Benjamin Jungfleisch, J. E. Pearson, X. Cheng, O. Heinonen, K. L. Wang, Y. Zhou, A. Hoffmann, and S. G. E. te Velthuis, Nat. Phys. 13, 162 (2016).

[21] K. Litzius, I. Lemesh, B. Krüger, P. Bassirian, L. Caretta, K. Richter, F. Büttner, K. Sato, O. A. Tretiakov, J. Förster, R. M. Reeve, M. Weigand, I. Bykova, H. Stoll, G. Schütz, G. S. D. Beach, and M. Kläui, Nat. Phys. 13, 170 (2016).

[22] D. Maccariello, W. Legrand, N. Reyren, K. Garcia, K. Bouzehouane, S. Collin, V. Cros, and A. Fert, Nat. Nanotechnol. 13, 233 (2018).

[23] K. Zeissler, S. Finizio, K. Shahbazi, J. Massey, F. Al Ma'Mari, D. M. Bracher, A. Kleibert, M. C. Rosamond, E. H. Linfield, T. A. Moore, J. Raabe, G. Burnell, and C. H. Marrows, Nat. Nanotechnol. 13, 1161 (2018).

[24] R. Tomasello, M. Ricci, P. Burrascano, V. Puliafito, M. Carpentieri, and G. Finocchio, AIP Adv. 7, 056022 (2017).

[25] A. Fert, V. Cros, and J. Sampaio, Nat. Nanotechnol. 8, 152 (2013).

[26] J. Sampaio, V. Cros, S. Rohart, A. Thiaville, and A. Fert, Nat. Nanotechnol. 8, 839 (2013).

[27] R. Tomasello, E. Martinez, R. Zivieri, L. Torres, M. Carpentieri, and G. Finocchio, Sci. Rep. 4, 6784 (2014).

[28] Y. Zhou, E. Iacocca, A. A. Awad, R. K. Dumas, F. C. Zhang, H. B. Braun, and J. Åkerman, Nat. Commun. 6, 8193 (2015).

[29] M. Carpentieri, R. Tomasello, R. Zivieri, and G. Finocchio, Sci. Rep. 5, 16184 (2015).

[30] G. Finocchio, M. Ricci, R. Tomasello, A. Giordano, M. Lanuzza, V. Puliafito, P. Burrascano, B. Azzerboni, and M. Carpentieri, Appl. Phys. Lett. 107, 262401 (2015).

[31] X. Zhang, M. Ezawa, and Y. Zhou, Sci. Rep. 5, 9400 (2015).

[32] D. Pinna, F. Abreu Araujo, J. V. Kim, V. Cros, D. Querlioz, P. Bessiere, J. Droulez, and J. Grollier, Phys. Rev. Appl. 9, 64018 (2018).

[33] D. Prychynenko, M. Sitte, K. Litzius, B. Krüger, G. Bourianoff, M. Kläui, J. Sinova, and K. Everschor-Sitte, Phys. Rev. Appl. 9, 014034 (2018).

[34] C. Schutte, J. Iwasaki, A. Rosch, and N. Nagaosa, Phys. Rev. B 90, 174434 (2014).

[35] R. Tomasello, K. Y. Guslienko, M. Ricci, A. Giordano, J. Barker, M. Carpentieri, O. Chubykalo-Fesenko, and G. Finocchio, Phys. Rev. B 97, 060402(R) (2018).

[36] R. Tomasello, A. Giordano, S. Chiappini, R. Zivieri, G. Siracusano, V. Puliafito, I. Medlej, A. La Corte, B. Azzerboni, M. Carpentieri, Z. Zeng, and G. Finocchio, Phys. Rev. B 98, 224418 (2018).

[37] B. F. McKeever, D. R. Rodrigues, D. Pinna, A. Abanov, J. Sinova, and K. Everschor-Sitte, Phys. Rev. B 99, 054430 (2019).

[38] M. Alonso and E. J. Finn, Fundamental University Physics, Vol. III (Addison-Wesley, Reading, MA, 1968).
[39] K. Huang, Statistical Mechanics, 2nd ed. (John Wiley \& Sons, New York, 1987).

[40] M. Ge, L. Zhang, D. Menzel, H. Han, C. Jin, C. Zhang, L. Pi, and Y. Zhang, J. Alloys Compd. 649, 46 (2015).

[41] H. Han, D. Menzel, W. Liu, L. Ling, H. Du, L. Pi, C. Zhang, L. Zhang, and Y. Zhang, Mater. Res. Bull. 94, 500 (2017).

[42] J. Wild, T. N. G. Meier, S. Pöllath, M. Kronseder, A. Bauer, A. Chacon, M. Halder, M. Schowalter, A. Rosenauer, J. Zweck, J. Müller, A. Rosch, C. Pfleiderer, and C. H. Back, Sci. Adv. 3, e1701704 (2017).

[43] See Supplemental Material at http://link.aps.org/supplemental/ 10.1103/PhysRevB.99.174440 for the details of the micromagnetic model and the calculation of the most probable value of skyrmion diameter population.

[44] A. Giordano, G. Finocchio, L. Torres, M. Carpentieri, and B. Azzerboni, J. Appl. Phys. 111, 07D112 (2012).

[45] V. Puliafito, A. Giordano, A. Laudani, F. Garescì, M. Carpentieri, B. Azzerboni, and G. Finocchio, Appl. Phys. Lett. 109, 202402 (2016).

[46] S. Rohart and A. Thiaville, Phys. Rev. B 88, 184422 (2013).

[47] R. Tomasello, M. Carpentieri, and G. Finocchio, J. Appl. Phys. 115, 17C730 (2014).

[48] W. F. Brown, Phys. Rev. 130, 1677 (1963).

[49] G. Finocchio, I. N. Krivorotov, X. Cheng, L. Torres, and B. Azzerboni, Phys. Rev. B 83, 134402 (2011).

[50] G. Finocchio, M. Carpentieri, E. Martinez, and B. Azzerboni, Appl. Phys. Lett. 102, 212410 (2013).

[51] M. Belmeguenai, J.-P. Adam, Y. Roussigné, S. Eimer, T. Devolder, J.-V. Kim, S. M. Cherif, A. Stashkevich, and A. Thiaville, Phys. Rev. B 91, 180405(R) (2015).

[52] H. Yang, A. Thiaville, S. Rohart, A. Fert, and M. Chshiev, Phys. Rev. Lett. 115, 267210 (2015).

[53] L. Zhang, B. Fang, J. Cai, M. Carpentieri, V. Puliafito, F. Garescì, P. K. Amiri, G. Finocchio, and Z. Zeng, Appl. Phys. Lett. 113, 102401 (2018).

[54] N. Mikuszeit, O. Boulle, I. M. Miron, K. Garello, P. Gambardella, G. Gaudin, and L. D. Buda-Prejbeanu, Phys. Rev. B 92, 144424 (2015).

[55] J. Barker and O. A. Tretiakov, Phys. Rev. Lett. 116, 147203 (2016).

[56] A. A. Belavin and A. M. Polyakov, Pisma Zh. Eksp. Teor. Fiz. 22, 503 (1975) [JETP Lett. 22, 245 (1975)].

[57] F. Tejo, A. Riveros, J. Escrig, K. Y. Guslienko, and O. Chubykalo-Fesenko, Sci. Rep. 8, 6280 (2018).

[58] D. Bazeia, D. C. Moreira, and E. I. B. Rodrigues, J. Magn. Magn. Mater. 475, 734 (2019).

[59] N. Romming, A. Kubetzka, C. Hanneken, K. von Bergmann, and R. Wiesendanger, Phys. Rev. Lett. 114, 177203 (2015).

[60] G. H. Wannier, Statistical Physics, 2nd ed. (Dover, New York, 1987).

Correction: A minor error in the definition for $\rho$ in third paragraph of Sec. III A has been fixed. A mislabeling in the citation for Eq. (B5) that appears in the second complete sentence after Eq. (B5) has been fixed. 